\section{Falsch positive Allergietests vermeidbar}

\author{
Falsch positive Tests in der Allergiediagnostik durch den Einfluss \\ kreuzreagierender Kohlenhydrat-Determinanten auf Proteinen etwa von \\ Pflanzen sind nicht selten. Sie können aber zum Beispiel durch Inhibitoren \\ der Determinanten vermieden werden.
}

$\mathrm{K}_{\mathrm{t} \text { ren }}^{\mathrm{r}}$ reuzreagierende Kohlenhydrat-Determinanten (CCD, ,cross-reactive carbohydrate determinants") lassen sich auf fast allen Pflanzenallergenextrakten und Insektengiften von Hymenopteren nachweisen. Sie sind häufig der Grund für falsch positive In-vitro-AllergieTests. Nach Angaben von Prof. Friedrich Altmann, Wien, liegt der Anteil der Allergiker, die IgE-Antikörper gegen diese CCDs entwickeln, zwischen $22 \%$ und $30 \%$. Bisher gebe es allerdings keine Belege dafür, dass CCDs allergische Reaktionen auslösen. Warum das so ist, ist noch unklar. Auch ist nicht bekannt, warum manche Menschen Anti-CCD-IgE entwickeln.

Um das Problem der falsch positiven Testergebnisse aufgrund der CCD zu umgehen, gibt es mehrere Möglichkeiten. Dazu gehören der Pricktest und der placebokontrollierte Nahrungsmittelprovokationstest. Eine andere Möglichkeit ist der Basophilenaktivierungstest in vitro. Allerdings werde dessen Ergebnis zumindest teilweise durch CCDs beeinflusst. Auch durch Verwendung rekombinanter Allergene in In-vitro-Tests lassen sich falsch positive Testergebnisse vermeiden. Der Grund: Die Allergene werden in der Regel mithilfe von Escherichia coli produziert und sind deshalb nicht glykosyliert. Altmann erinnert aber daran, dass es Testsysteme mit mehreren Testkomponenten gibt, in denen auch Allergene aus natürlichen Extrakten enthalten sind.

Schließlich lässt sich der Einfluss der CCDs auf das Testergebnis dadurch beeinflussen, dass CCD-spezifische Inhibitoren bei der In-vitro-Testung verwendet werden. Dazu kann jegliches Pflanzenglykoprotein genutzt werden, das eine MMXF- oder eine MUXF-Struktur aufweist: Beides sind N-Glykane mit $\alpha-1,3$-gebundener Fucose, die im gesamten Pflanzenreich und bei Insekten vorkommen, nicht dagegen bei Säugetieren.

Um den multivalenten Effekt nutzen zu können, sollten solche als Inhibitoren verwendeten Glykoproteine mehrere CCD-Strukturen enthalten. Als Beispiel nennt Altmann einen Cocktail, der aus den drei Glykoproteinen Bromelain, Meerrettichperoxidase und Ascorbatoxidase besteht. Es gibt auch CCD-Inhi- bitoren, die zuvor mit dem Enzym Protease behandelt werden, wie ein Proglykan-CCD-Blocker aus Bromelain. Die durch die Aktivität des Enzyms entstehenden Glykopeptide werden anschließend gereinigt und an Albumin gekoppelt. Dadurch wird das Risiko von Peptid-IgE-Wechselwirkungen minimiert, die zu unerwünschter Signalsenkung führen würden. Die CCD-Hemmung hat bislang in mehreren Testsystemen zu einer deutlichen Reduktion falsch positiver Testergebnisse geführt. Peter Leiner

Altmann F. Coping with cross-reactive carbohydrate determinants in allergy diagnosis. Allergo J Int 2016; 25: 98-105

\section{Kommentar:}

Jeder der sich mit IgE-In-vitro-Diagnostik befasst kennt die Problematik der kreuzreagierenden Kohlenhydrat-Determinanten. Die vorliegende Arbeit beschreibt eine einfache und elegante Lösung wie man dem CCD-Problem Herr werden kann. Besonders für Leser, die selber ein Allergielabor betreiben, eine unbedingt empfehlenswerte Lektüre.

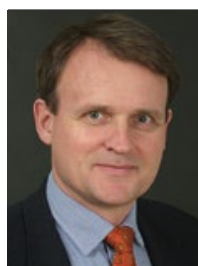

Prof. Dr. Thilo Jakob Direktor der Klinik für Dermatologie und Allergologie Universitätsklinikum Gießen u. Marburg, Standort Gießen

\section{Basophilenaktivierungstest: Provokation auf zelluärer Ebene}

Der Basophilenaktivierungstest ist dabei, sich sowohl in der Diagnostik als auch beim Therapie-Monitoring einen Platz zu erobern. Bis der Test in der Laborroutine ankommt muss er allerdings noch ein paar Hürden nehmen.

$\mathrm{N}$ eben IgE-Bestimmung, Haut-Pricktest und Intradermaltest sowie im Vorfeld von Provokationstests könnte der Basophilenaktivierungstest (BAT) schon bald Teil der Allergiediagnostik werden. Sein Einsatz lohnt sich vor allem dann, wenn für Testallergene von Hauttests häufig falsch positive Ergebnisse bekannt sind, kein Allergen für
Haut- oder IgE-Tests zur Verfügung steht, sich Unstimmigkeiten zwischen Anamnese und IgE- oder Hauttests zeigen oder bei Letzteren eine systemische Reaktion befürchtet wird. Aber auch zum Monitoring während einer Hyposensibilisierung oder einer Therapie mit monoklonalem Anti-IgE oder zur Überprüfung einer Toleranzentwick- lung kann der BAT herangezogen werden.

Das Testprinzip beruht auf der In-vitro-Aktivierung basophiler Granulozyten durch ein breites Spektrum von Allergenen. Gemessen wird diese als verstärkte Expression bestimmter Aktivierungsmarker auf der Oberfläche der Basophilen im Rahmen einer allergischen Reaktion vom Soforttyp. Der derzeit klinisch am besten validierte Test basiert auf dem Oberflächenprotein CD63. Aber auch Testverfahren, die CD203c als Marker nutzen, haben sich als zuverlässig erwiesen.

Läuft nach Zugabe der zu testenden Antigene zum Patientenblut eine allergische Reaktion ab und wird durch die 